\title{
Synchrotron Superbubble in the Galaxy IC 10: The lonized Gas Structure, Kinematics, and Emission Spectrum
}

\author{
T.A. Lozinskaya ${ }^{1}$, A.V. Moiseev $^{2}$, N.Yu. Podorvanyuk ${ }^{1}$, and A.N. Burenkov ${ }^{2}$ \\ 1 Sternberg Astronomical Institute, Universitetskii pr. 13, Moscow, 119992 Russia \\ 2 Special Astrophysical Observatory RAS, Nizhnii Arkhyz, Karachai-Cherkessian Republic, 357147 Russia
}

the date of receipt and acceptance should be inserted later

\begin{abstract}
We have investigated the structure, kinematics, and emission spectrum of the ionized gas in the synchrotron superbubble in the irregular galaxy IC 10 based on observations with the 6-m Special Astrophysical Observatory telescope with the SCORPIO focal reducer in three modes: direct imaging in the $[\mathrm{SII}] \lambda(6717+6731) \AA$ lines, long-slit spectroscopy, and spectroscopy with a scanning Fabry-Perot interferometer. We have identified a bright (in the [SII] lines) filamentary optical shell and determined its expansion velocity, mass, and kinetic energy. The nature of the object is discussed.
\end{abstract}

Key words. interstellar medium, supernova remnants, kinematics and dynamics of galaxies, the galaxy IC 10.

\section{Introduction}

The dwarf irregular galaxy IC 10 is widely used to investigate the structure, kinematics, and emission spectrum of the interstellar medium in starburst regions. Multiple ionized and neutral shells and supershells, arc and ring structures with sizes from 50 to $800-1000$ pc, and a diffuse ionized gas component are observed in this galaxy (Zucker 2000; Wilcots and Miller 1998; Gil de Paz et al. 2003; Leroy et al. 2006; Chyzy et al. 2003; and references therein). Its stellar population suggests a recent starburst $(t=4-10 \mathrm{Myr})$ and an older starburst $(t>350 \mathrm{Myr})$ (Hunter 2001; Zucker 2002; Massey et al. 2007).

Two features distinguish IC 10 among other dwarf starburst galaxies and make its studies particularly interesting:

(1) The anomalously high number of Wolf-Rayet (WR) stars, which is a factor of 20 greater than that in the Large Magellanic Cloud. The space density of WR stars reaches 11 per square kiloparsec (Massey et al. 1992; Richer et al. 2001; Massey and Holmes 2002; Crowther et al. 2003; Massey et al. 2007; Vacca et al. 2007; and references therein). This is the highest density of WR stars among the dwarf galaxies comparable to that in massive spiral galaxies. For a normal initial mass function, such a high density of WR stars is indicative of an almost "simultaneous" current starburst affecting the bulk of the galaxy.

Send offprint requests to: T.A. Lozinsksaya, e-mail: lozinsk@sai.msu.ru
(2) The so-called synchrotron superbubble discovered by Yang and Skillman (1993) and, as it seems to us, has not yet been explained exhaustively. The authors associated this extended source of nonthermal radio emission with the explosions of about ten supernovae. Given that the characteristic detectability time of the synchrotron radio emission from a supernova remnant is $\sim 10^{5} \mathrm{yr}$, these explosions should also have occurred almost simultaneously. The model of multiple supernova explosions is also used in present-day papers (see, e.g., Bullejos and Rosado 2002; Rosado et al. 2002; Thurow and Wilcots 2005). Recently Lozinskaya and Moiseev (2007) offered an alternative explanation for the nature of the synchrotron superbubble: a hypernova explosion.

The synchrotron nature of the radio emission from the superbubble is confirmed by the high degree of its polarization revealed by Chyzy et al. (2003). Figure 2 from the above paper shows that the polarization vector is parallel to the dense dust layer observed here.

Note that, in fact, the observations by Yang and Skillman (1993) reveal no shell structure of this radio source, so the term "superbubble" was introduced by the authors based on the nature of the object suggested by them.

In this paper, we investigate in detail the structure, kinematics, and emission spectrum of the ionized gas in the region of the nonthermal radio source based on observations with the 6-m Special Astrophysical Observatory (SAO) telescope with the SCORPIO focal reducer in three modes (direct [SII] line imaging, long-slit spectroscopy, and spectroscopy with a scanning Fabry-Perot interfer- 
ometer). The data obtained revealed a bright (in [SII] lines) filamentary shell that could be identified with the synchrotron superbubble. We determined the expansion velocity, mass, and kinetic energy of the optical shell and discuss the nature of the object.

In the succeeding sections, we describe the observing and data reduction techniques, present and discuss the results obtained, and summarize our main conclusions.

The published estimates of the color indices and distance for IC 10 vary greatly due to the low Galactic latitude of the object, $b=3^{\circ} .3$ (Demers et al. 2004). In our paper, we use the values of $E(B-V)=0.95^{m}$ and $(m-M)_{0}=24.48 \pm 0.08^{m}$ refined by Vacca et al. (2007), corresponding to a distance of $r=790 \mathrm{kpc}$ to IC 10 and an angular scale of $\simeq 4 \mathrm{pc} /{ }^{\prime \prime}$.

All of the radial velocities in this paper are heliocentric.

\section{Observations and data reduction}

\subsection{Interferometric Observations}

Interferometric observations of IC 10 were performed on September 8/9, 2005, at the prime focus of the 6-m telescope using the SCORPIO focal reducer; the equivalent focal ratio of the system was $F / 2.6$. SCORPIO was described by Afanasiev and Moiseev (2005) and on the Internet (http://www.sao.ru/hq/lsfvo/devices.html); the SCOPRIO capabilities in interferometric observations were also described by Moiseev (2002).

We used a scanning Fabry-Perot interferometer (FPI) operating in the 501st order at the $\mathrm{H} \alpha$ wavelength. The spacing between the neighboring orders of interference, $\Delta \lambda=13 \AA$, corresponded to a region free from order overlapping of $\sim 600 \mathrm{~km} \mathrm{~s}^{-1}$ on the radial velocity scale. The FWHM of the instrumental profile was $\sim 0.8 \AA$, or $\sim$ $35 \mathrm{~km} \mathrm{~s}^{-1}$. Premonochromatization was performed using an interference filter with $\mathrm{FWHM}=13 \AA$ centered on the $\mathrm{H} \alpha$ line. The detector was an EEV 42-40 $2048 \times 2048$ pixel CCD array. The observations were performed with $2 \times 2$-pixel binning to reduce the readout time. In each spectral channel, we obtained $1024 \times 1024$-pixel images at a scale of $0.35^{\prime \prime}$ per pixel; the total field of view was $6.1^{\prime}$.

During our observations, we successively took 36 interferograms of the object for various FPI plate spacings, so the width of the spectral channel corresponded to $\delta \lambda=0.37 \AA$, or $17 \mathrm{~km} \mathrm{~s}^{-1}$ near $\mathrm{H} \alpha$. To properly subtract parasitic ghosts from the galaxy's numerous emission regions, the observations were performed for two different orientations of the instrument's field of view. The total exposure time was $10800 \mathrm{~s}$; the seeing (the FWHM of field-star images) varied within the range 0 ". $8-1$." 3 .

We reduced the observations using software running in the IDL environment (Moiseev 2002). After the primary reduction, the observational data were represented as $1024 \times 1024 \times 36$ data cubes; here, a 36 -channel spectrum corresponds to each pixel. The final angular resolution (after smoothing during the data reduction) was $\sim 1^{\prime \prime} .2$. The formal accuracy of the constructed wavelength scale was about $3-5 \mathrm{~km} \mathrm{~s}^{-1}$.

\subsection{Images in Emission Lines}

Images in the [SII] $\lambda 6717,6731 \AA$ doublet emission lines were obtained on the 6 - $\mathrm{m}$ telescope with the SCORPIO focal reducer. A log of photometric observations is given in Table 1, which lists the dates of observations, the central wavelengths $\left(\lambda_{c}\right)$ and FWHMs of the filter used, the total exposure times $\left(T_{\exp }\right)$, and the average seeing. This table includes a filter whose passband contained the [SII] $\lambda 6717,6731 \AA$ emission lines and a filter centered on the continuum near these lines.

The detector was an EEV42-40 CCD that provided a scale of $0.36^{\prime \prime}$ per pixel (in $2 \times 2$ readout mode). The data were reduced using a standard procedure. When constructing the maps of "pure" [SII] line emission, we subtracted the continuum image from the images in the filter containing the emission lines and continuum.

A monochromatic image in $\mathrm{H} \alpha$ was constructed by integrating the flux in this emission line in the FPI spectra (see the previous section).

\subsection{Long-Slit Spectroscopy}

Spectroscopic observations of IC 10 were performed with the same SCORPIO instrument operating in the mode of a long-slit spectrograph with a slit about $6^{\prime}$ in length and $1^{\prime \prime}$ in width. The scale along the slit was $0.36^{\prime \prime}$ per pixel. The total exposure time was $3600 \mathrm{~s}$ at a seeing of about 1 .' 4 . We used the volume phase holographic grating VPHG1200R, which provided the spectral range $\Delta \lambda=$ 5650-7350 $\AA$ that included the $\mathrm{H} \alpha$, [NII] $\lambda 6548,6583 \AA$, and [SII] $\lambda 6717,6731 \AA$ emission lines. The spectral resolution estimated from the widths of night-sky lines was about $6 \AA$. The data were reduced in a standard way; the spectrophotometric standard BD $+25 d 4655$ observed immediately after the object almost at the same zenith distance was used for energy calibration. The intensities and radial velocities of the emission lines were determined by means of single-component Gaussian fitting. The accuracy of the absolute velocity measurements estimated from night-sky lines was $\sim 10-20 \mathrm{~km} \mathrm{~s}^{-1}$.

\section{Results of observations}

\subsection{Morphology of the Region in the $\mathrm{H} \alpha$ and [SII] Lines}

Figure 1 shows our $[\mathrm{SII}] \lambda(6717+6731) \AA$ line image of the southeastern part of IC 10 with 20 -cm radio emission contours from Yang and Skillman (1993) superimposed. The synchrotron superbubble is located southeast of the dust lane that is clearly seen in the galaxy's optical images.

Figure 2 compares the $\mathrm{H} \alpha$ and [SII] line images of the region. Figure 2a shows the synchrotron superbubble region in $\mathrm{H} \alpha$ based on our FPI observations. Also shown 
Table 1. Log of photometric observations

\begin{tabular}{l|c|c|c|c|c}
\hline \multicolumn{1}{c|}{ Range } & Date & $\lambda_{c}, \AA$ & FWHM, $\AA$ & $T_{\exp , \mathrm{s}}$ & Average seeing, " \\
\hline [SII] & Feb. 14/15, 2007 & 6740 & 50 & 5600 & $1.1-1.5$ \\
[SII] continuum & Feb. 14/15, 2007 & 7040 & 210 & 1650 & $1.1-1.5$ \\
\hline
\end{tabular}

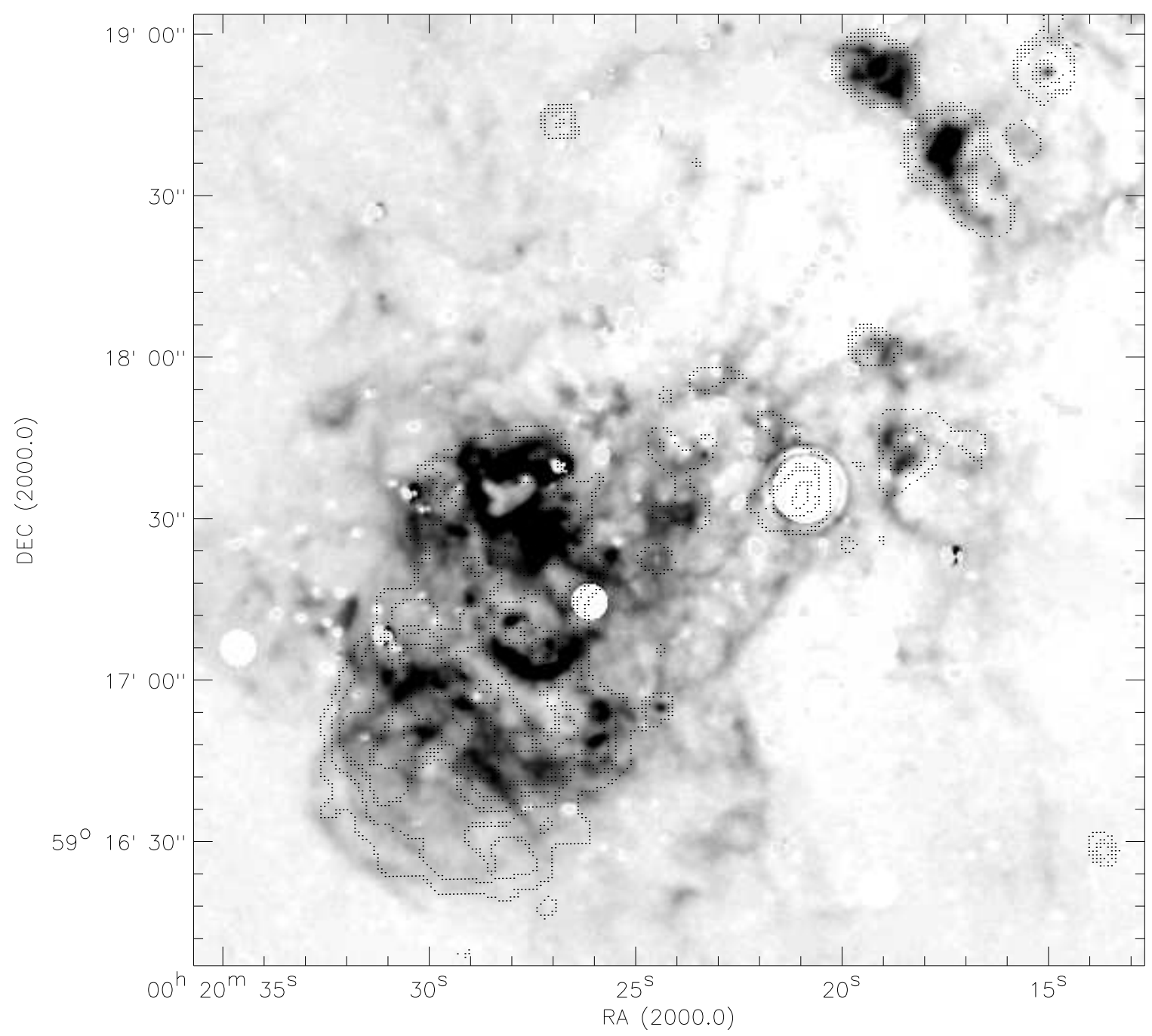

Fig. 1. $[\mathrm{SII}] \lambda(6717+6731) \AA$ lines image of the southeastern part of the galaxy obtained with the 6 -m telescope with 20-cm radio emission contours from Yang and Skillman (1993) superimposed.

here are the positions of spectroscopically confirmed WR stars from the lists by Royer et al. (2001) and Massey and Holmes (2002) as well as star clusters in the region from the list by Hunter (2001). Figure 2b presents our image of the region in the sulfur emission lines. The [SII] doublet lines are known to be optimal for revealing the emission from the gas behind the front of a shock propagating with a velocity characteristic of old supernova remnants. Indeed, a filamentary shell about $40^{\prime \prime}-44^{\prime \prime}$ in size with the center at $\alpha_{(2000)}=00_{20} \mathrm{~m}_{29^{\mathrm{S}}}, \delta_{(2000)}=59^{\circ} 16^{\prime} 40^{\prime \prime}$ is seen most clearly in the [SII] line image of the galaxy.

The shell is morphologically inhomogeneous in the plane of the sky: a brighter diffuse symmetric spherical structure about $30^{\prime \prime}$ in size with the center at $\alpha_{(2000)}=$ ${ }_{0} \mathrm{~h}_{20} \mathrm{~m}_{30^{\mathrm{s}}}, \delta_{(2000)}=59^{\circ} 16^{\prime} 44^{\prime \prime}$ and a filamentary struc- ture about $44^{\prime \prime} \times(30-40)^{\prime \prime}$ in size with the center at $\alpha_{(2000)}=0^{\mathrm{h}} 20 \mathrm{~m}_{30^{\mathrm{S}}}, \delta_{(2000)}=59^{\circ} 16^{\prime} 36^{\prime \prime}$ elongated in the same direction as the dust layer are noticeable in the [SII] lines. These can be a dumbbell-type structure or an aspherical shell with a nonuniform brightness. Both variants can be naturally explained by a nonuniform density of the ambient interstellar medium, as evidenced by the presence of a dense $\mathrm{HI}$ and $\mathrm{CO}$ cloud (see the text below) and a dust layer. This optical shell structure, which is bright in the [SII $] \lambda(6717+6731) \AA$ lines, agrees in localization and size with the the radio shell (see Fig. 1). Therefore, it can be identified with the optical emission from the synchrotron superbubble.

At least three more fainter filamentary shell structures that go far beyond the synchrotron superbubble are ob- 
a)

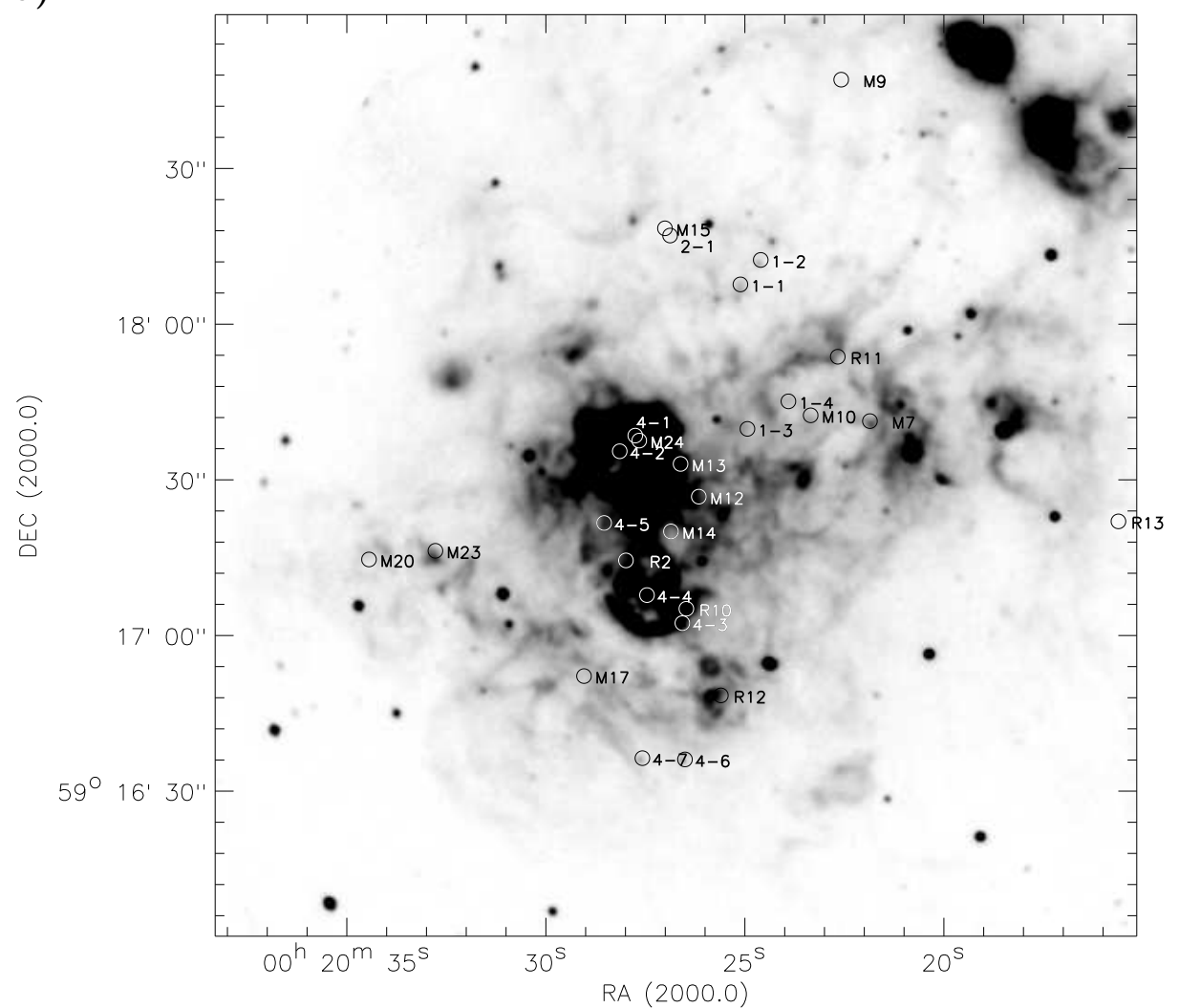

a)

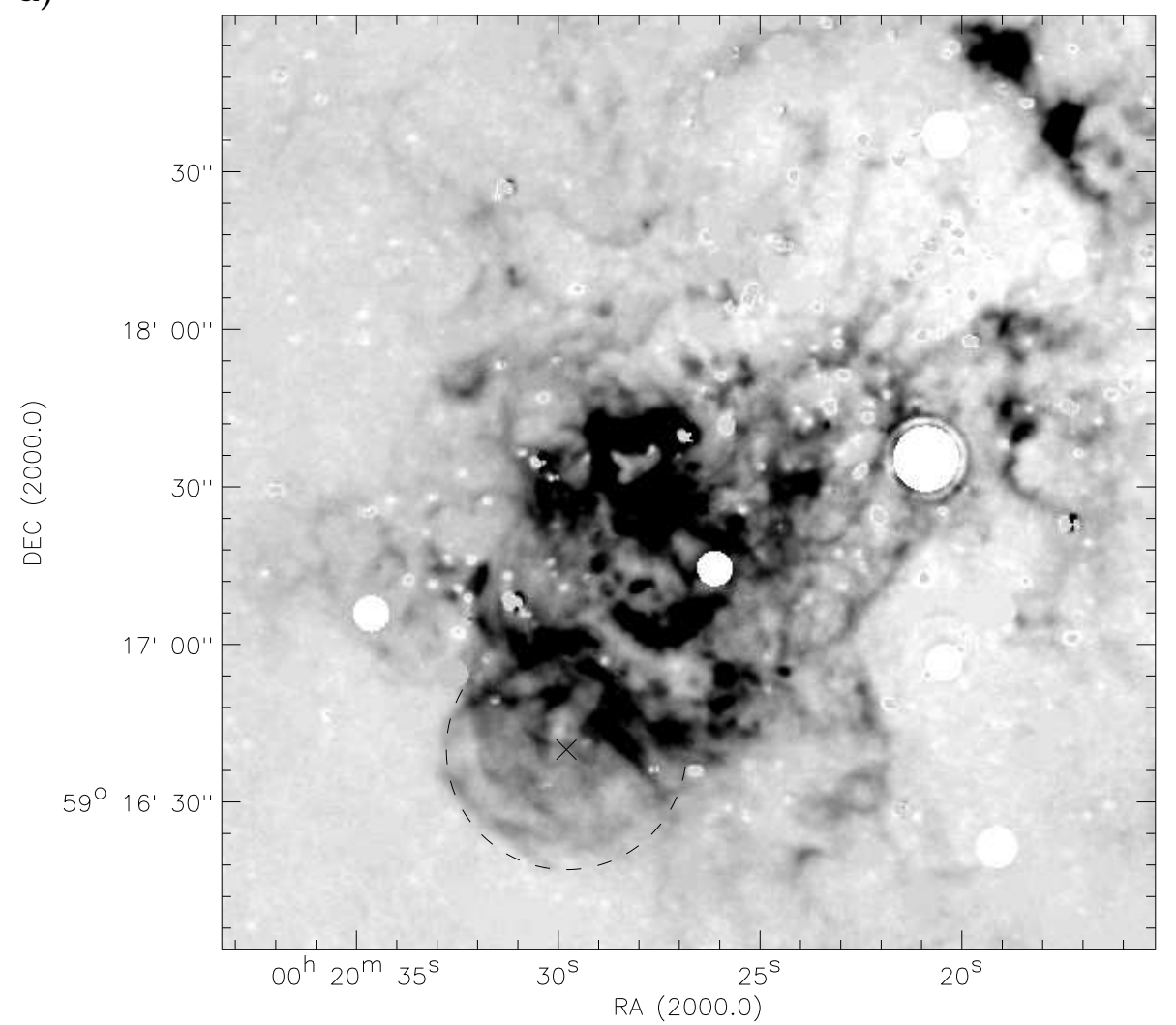

Fig. 2. Images of the synchrotron superbubble region (a) in $\mathrm{H} \alpha$ based on our FPI observations and (b) in the [SII] $\lambda(6717+6731) \AA$ lines (the continuum was subtracted). The positions of spectroscopically confirmed WR stars from the lists by Royer et al. (2001) (denoted by the letter R) and Massey and Holmes (2002) (denoted by the letter M) as well as star clusters from the list by Hunter (2001) (denoted by two numerals) are shown. 
a)
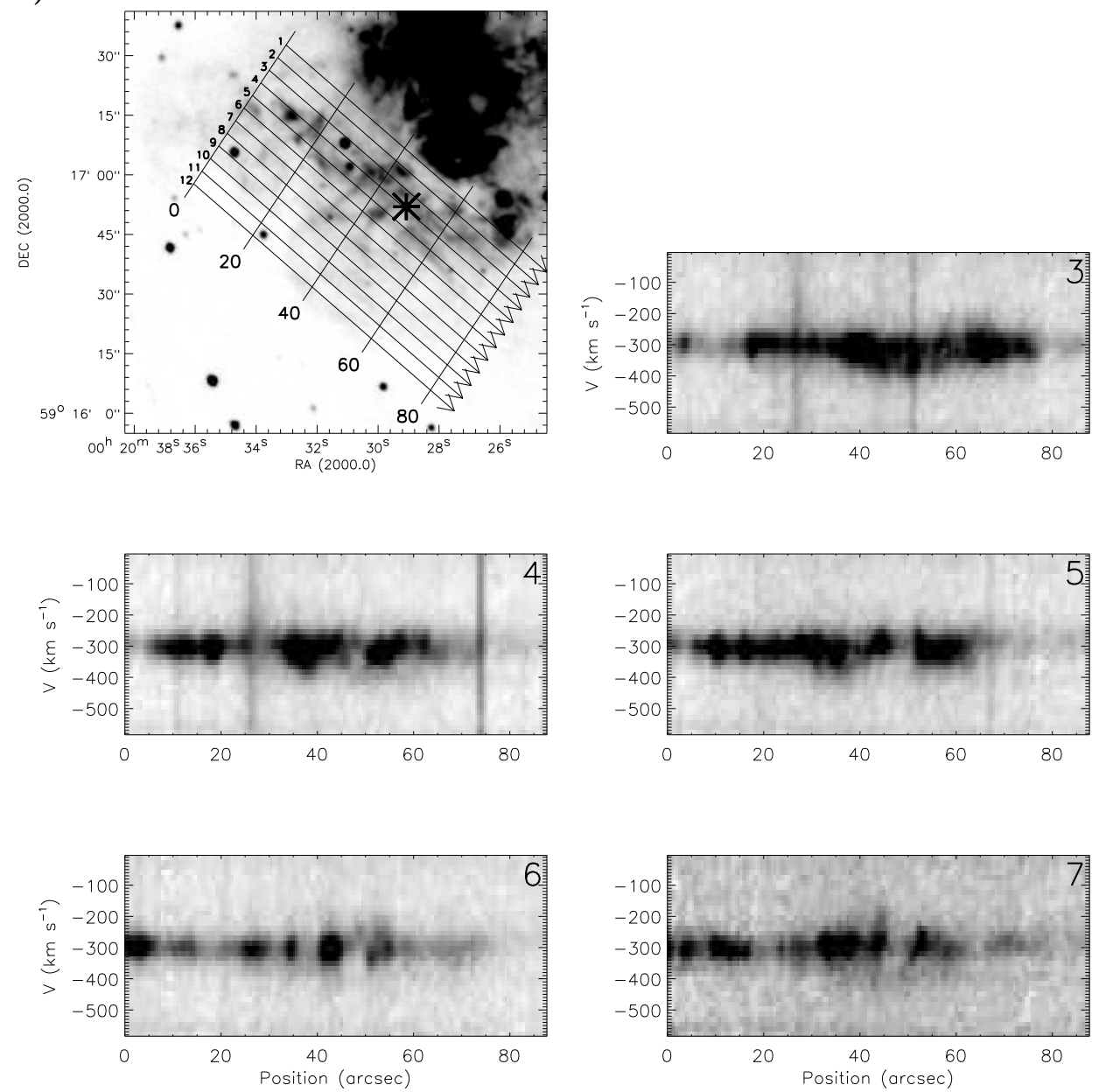

a)
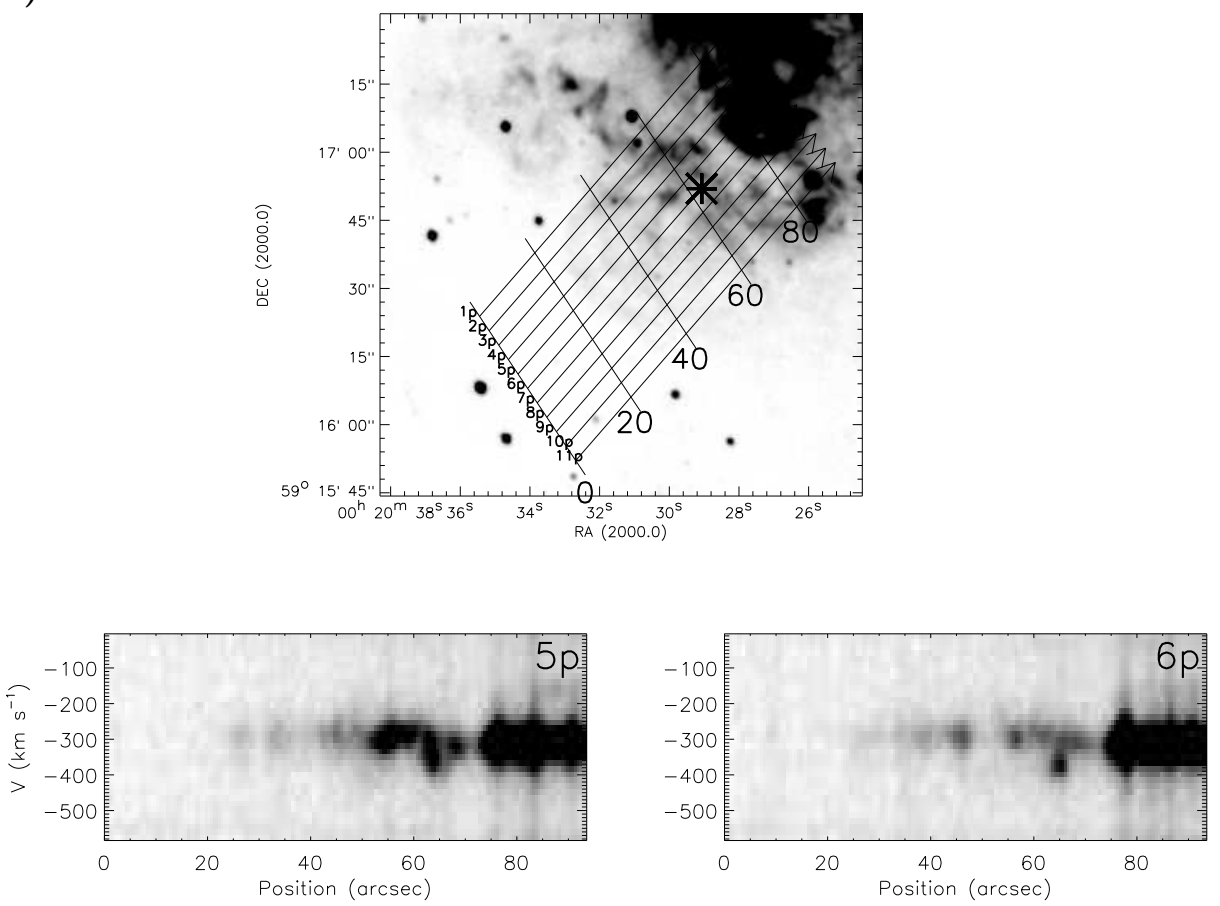

Fig. 3. Localization of the two systems of scans for which the $P / V$ diagrams were constructed from our FPI observations in $\mathrm{H} \alpha$ and some of the $P / V$ diagrams. The scans are marked up in arsec: (a) 12 scans in directions parallel to the dust layer; (b) 11 scans in a perpendicular direction. The asterisk marks the position of the star WR M17. 

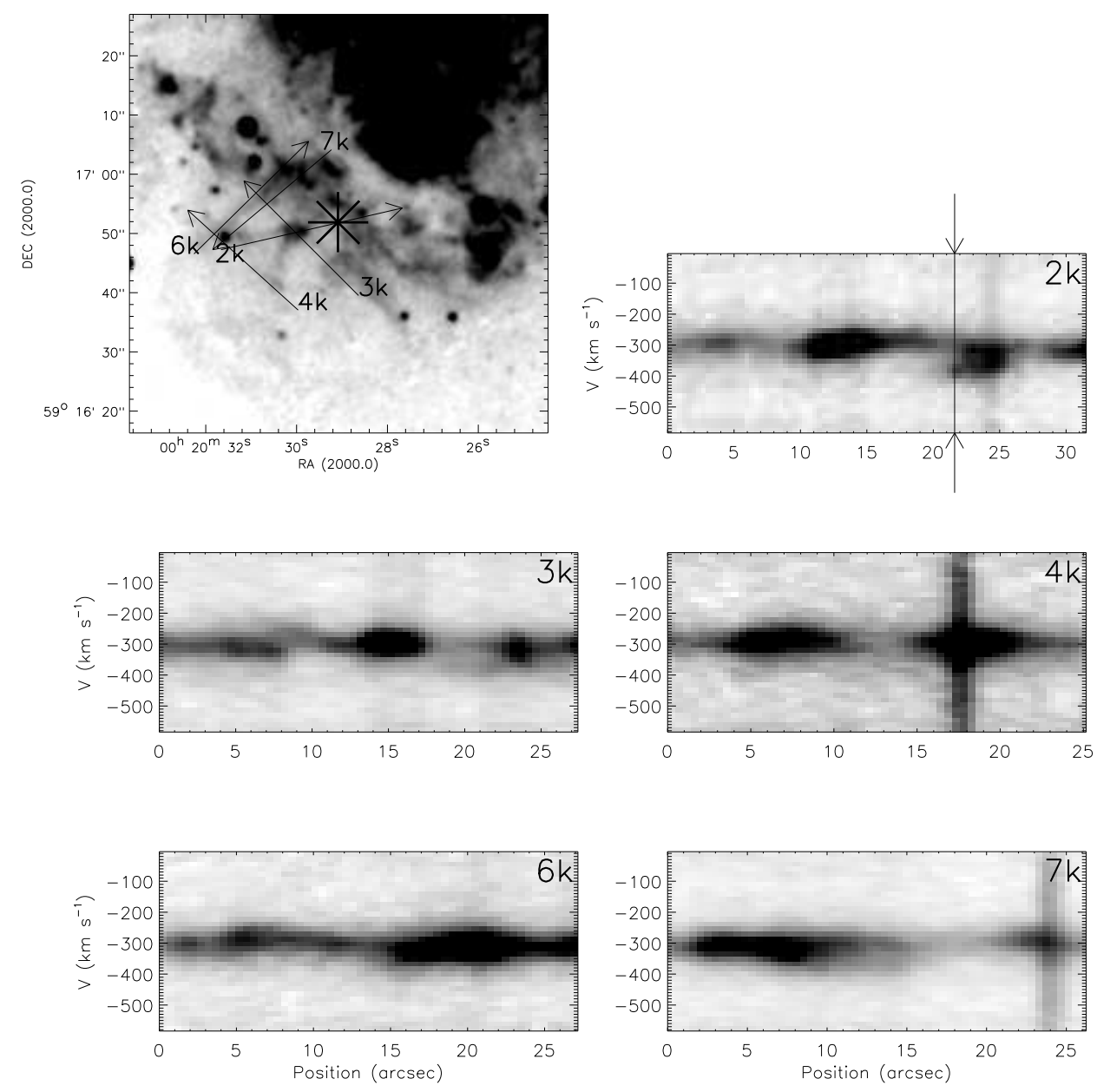

Fig. 4. Localization of several scans and the corresponding $P / V$ diagrams in the vicinity of the $\mathrm{X}$-ray source $\mathrm{X}-1$, a black hole in a pair with the WR star M17. The arrows on the diagram 2k indicate the star WR M17 location.

served northeast, west, and southwest of this bright optical shell.

The [SII] line image of the galaxy from Rosado et al. (1999) is also indicative of an intense emission in the region. We do not associate the arc structure far beyond the western boundary of the radio source that was mentioned by these authors with the synchrotron superbubble, because there is no detectable radio emission here.

As we see, the optical emission is characterized by a filamentary structure both in the synchrotron superbubble and in its immediate vicinity. The wind from at least four WR stars can be important in producing the filamentary structure of the region: M20 and M23 are located in the "northeastern" shell, M17 is located in the shell identified with the synchrotron radio source; R12 is located in the bright part of the "western" shell. The star M17 is currently believed to be a component of an X-ray binary: a WR star in a pair with a black hole - the brightest Xray source X-1 in the Galaxy (Brandt et al. 1997; Bauer and Brandt 2004; Wang et al. 2005). As we showed in (Lozinskaya and Moiseev 2007), this object can represent the compact remnant of a hypernova whose explosion gave rise to the synchrotron superbubble.
The contribution of two star clusters, 4-6 and 4-7, from the list by Hunter (2001) can also be important in producing multiple shells in this region of the galaxy.

\subsection{Ionized Gas Kinematics in the Synchrotron Superbubble}

Based on our $\mathrm{H} \alpha$ observations with a scanning FPI, we investigated the kinematics of the ionized hydrogen in the entire region southeast of the dust layer. Taking into account the complex multishell filamentary structure of the region, the presence of six stellar wind sources mentioned above, and the possible presence of several supernova explosions, we investigated this region in greatest detail. Diagrams of the gas radial velocity distribution (the so-called position-velocity or $P / V$ diagrams) were constructed along 45 scans crossing the synchrotron superbubble in various directions.

Figures $3 \mathrm{a}$ and $3 \mathrm{~b}$ show the localization of 12 scans in directions parallel to the dust layer and 11 scans in a perpendicular direction. For the convenience of orientation, the scans in each figure are marked up in arcsec. Also shown here are some of the $P / V$ diagrams constructed for 

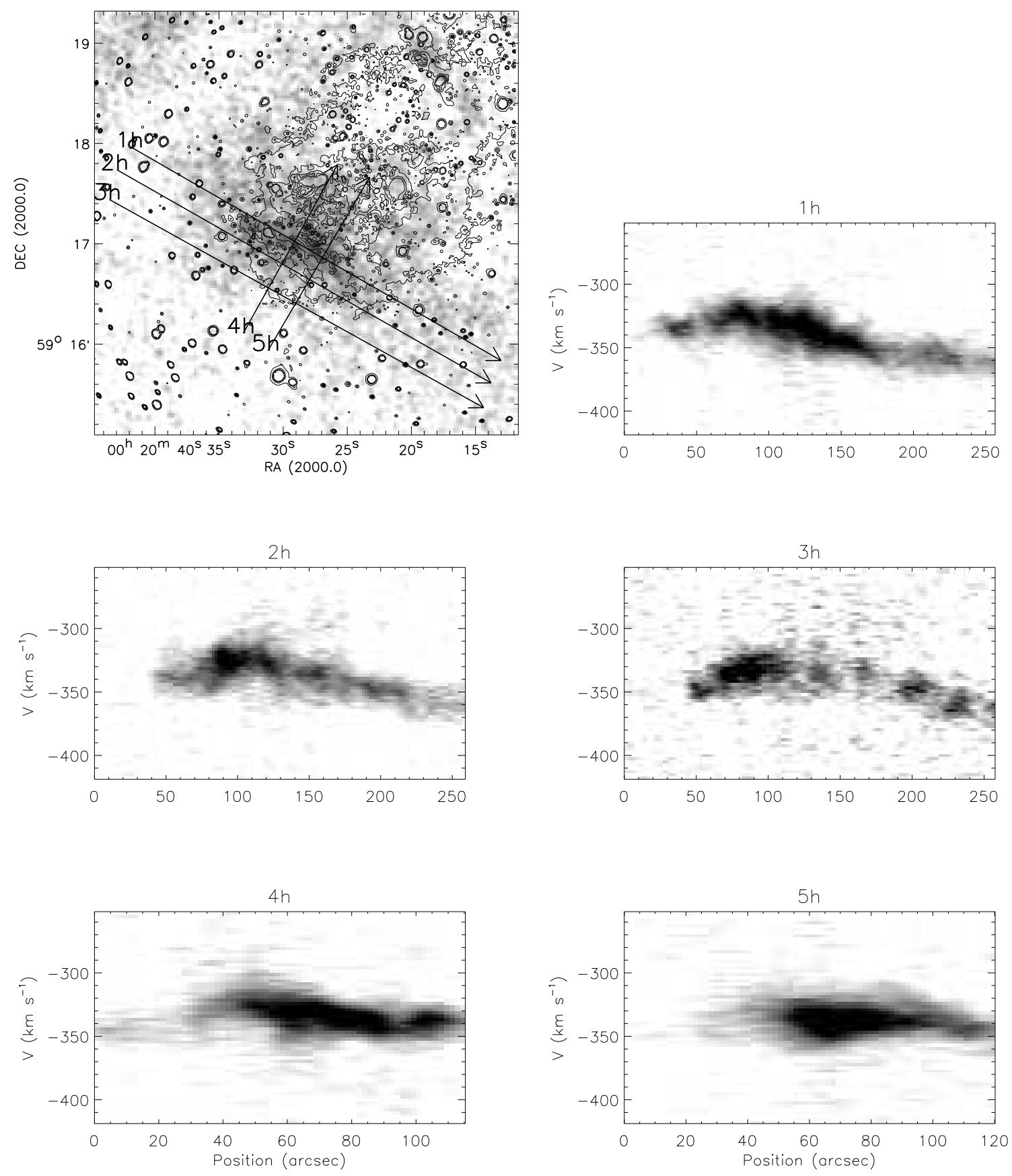

Fig. 6. Localization of the HI scans passing through the synchrotron superbubble and the $P / V$ diagrams that we constructed from the 21-cm observations by Wilcots and Miller (1998). The shades of gray indicate the HI brightness distribution; the isophotes indicate the $[\mathrm{SII}] \lambda(6717+6731) \AA$ line image.

these scans that allow high-velocity motions to be revealed in the region.

The mean gas velocity in the galactic region under consideration was estimated from our FPI $\mathrm{H} \alpha$ observations to be about $-300--310 \mathrm{~km} \mathrm{~s}^{-1}$; we revealed a radial velocity gradient from the northeast to the southwest up to $-320--330 \mathrm{~km} \mathrm{~s}^{-1}$. This is in agreement with the data from Thurow and Wilcots (2005) (-315--320 $\mathrm{km} \mathrm{s}^{-1}$, as inferred from $\mathrm{H} \alpha$ observations) and the data from Wilcots and Miller (1998) $\left(-326 \mathrm{~km} \mathrm{~s}^{-1}\right.$, as inferred from 21-cm HI observations).
Our $P / V$ diagrams do not reveal the classical velocity ellipse (the radial velocities of the near and far sides of an expanding shell decrease in absolute value along the radius in the plane of the sky). This can be naturally explained by the inhomogeneity of the synchrotron superbubble, which is actually represented by knots and filaments both in $\mathrm{H} \alpha$ and in the [SII] lines (see Fig. 2).

At the same time, we see distinct deviations of the velocities of ionized gas clouds and filaments from the derived mean gas velocity in the shell. Scans nos. 3 (positions $45-65$ arcsec), 4 (37-57 arcsec), 5 (37-53 arcsec), 6 

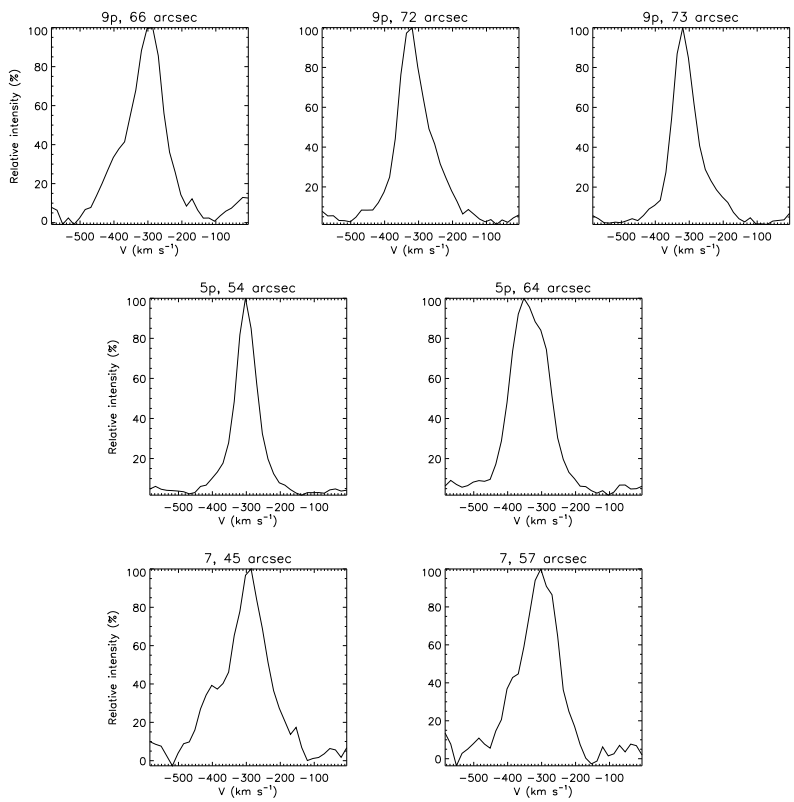

Fig. 5. $\mathrm{H} \alpha$ profiles for scans nos. $9 \mathrm{p}$ (in a region of 66,72 , and 73 arcsec), 5p (54 and 64 arsec), and 7 (45 and 57 arcsec) shown in Fig. 3.

(45-53 arcsec), and 7 (about 47 arcsec) as well as nos. 5p (61-67 arcsec) and 6p (61-67 arcsec) presented in Fig. 3 demonstrate the largest velocity deviations.

As we see, all these scans pass through the central part of the superbubble near the brightest X-ray source $\mathrm{X}-1$ in the galaxy, a black hole in a pair with the WR star M17. Since this object is an obvious source of kinetic energy that affects the gas structure and kinematics in the synchrotron superbubble, more than 10 additional scans were made in its immediate vicinity on a larger scale. The localization and corresponding $P / V$ diagrams of several of them are shown in Fig. 4.

The gas kinematics near all WR stars in the galaxy will be investigated in detail in our next paper (Lozinskaya et al. 2008). Therefore, we do not consider here the three more WR stars mentioned above that are located in the northeast and the southwest outside the synchrotron superbubble.

As follows from Fig. 4, the star M17 is located in a local aspherical cavity elongated from the north to the south. The distance to the outer boundaries of the cavit $\mathrm{y}$ is about $2^{\prime \prime}$ north, east, and west of the star and reaches 5 $6^{\prime \prime}$ in the southeast ( 8 and $19-23 \mathrm{pc}$, respectively). The largest deviations of the mean radial velocity from a characteristic value of $-300 \mathrm{~km} \mathrm{~s}^{-1}$ in the superbubble region are observed in the bright walls of this cavity: about $-360--380 \mathrm{~km} \mathrm{~s}^{-1}$ on scans nos. $2 \mathrm{k}, 4 \mathrm{k}$, and $6 \mathrm{k}$. The mean velocities of the features with a positive shift relative to the mentioned characteristic velocity reach -280 $-250 \mathrm{~km} \mathrm{~s}^{-1}$ (see scans nos. $2 \mathrm{k}$ - position $14 \operatorname{arcsec}, 3 \mathrm{k}-$ 8 and 15 arcsec, $6 \mathrm{k}-20-22$ arcsec, and $7 \mathrm{k}-15$ arcsec). These features are in the shape of bridges in the $P / V$ dia- grams and correspond to low-brightness regions inside the cavity.

We emphasize that the velocities mentioned above were determined from the line peak, i.e., they characterize the brightest knots and filaments. We estimated the expansion velocity of the superbubble determined by the deviations of the line peak velocity from its mean value in the bright northern part of the nebula to be about 50 $80 \mathrm{~km} \mathrm{~s}^{-1}$. Fainter wings at a level of about $20-30 \%$ of the peak intensity are observed in the synchrotron superbubble region in the velocity range from $-450--420$ to $-200--240 \mathrm{~km} \mathrm{~s}^{-1}$.

The $\mathrm{H} \alpha$ profiles for scans nos. 9p (in a region of 66 , 72 , and 73 arcsec), 5p (54 and 64 arcsec), and 7 (45 and 57 arcsec) presented in Fig. 5 can serve as an example. As we see from the figure, some of these profiles exhibit a double-peaked structure.

\subsection{Neutral Gas Kinematics in the Synchrotron Superbubble}

Based on 21-cm line observations, Wilcots and Miller (1998) investigated in detail the kinematics of the neutral hydrogen in the galaxy. We reanalyzed the data cube kindly provided by these authors to present the results in a form comparable to our studies of the ionized gas kinematics. Our results completely confirmed the data from the above paper. Figure 6 shows the localization of five scans passing through the synchrotron superbubble for which we constructed the $P / V$ diagrams for the neutral gas. As follows from the figure, the neutral hydrogen in the region of the synchrotron radio source clearly reveals kinematic signatures of the shell expansion - the classical picture of half the velocity ellipse corresponding to the receding side of the shell. We emphasize that the shell structure in the neutral hydrogen distribution in the region of the synchrotron radio source is unseen; we are talking about an expanding shell only based on the gas kinematics. The mean expansion velocity of the HI shell is $25-30 \mathrm{~km} \mathrm{~s}^{-1}$. The mass of the neutral gas drawn into this expansion reaches $M \simeq 7 \times 10^{5} M_{\odot}$, as estimated by Wilcots and Miller (1998) (the authors point out that this value is determined with a low accuracy). The corresponding kinetic energy is $E_{\text {kin }} \simeq 5 \times 10^{51} \mathrm{erg}$.

The vicinities of the synchrotron superbubble are the dynamically most active region of the galaxy in the $21-\mathrm{cm}$ line: such a distinct velocity ellipse is observed nowhere and the possible expansion velocity of the local HI shells in IC 10 estimated by Wilcots and Miller (1998) nowhere exceed $20 \mathrm{~km} \mathrm{~s}^{-1}$.

\subsection{The Gas Emission Spectrum in the Synchrotron Superbubble}

The localization of the slit spectrogram passing through the synchrotron superbubble is shown in Fig. 7. For the convenience of identification, the spectrogram in the fig- 
ure is marked up in arcsec; the region from $90^{\prime \prime}$ to $125^{\prime \prime}$ corresponds to the superbubble.

The spectrum processing results are presented in Fig. 8; when analyzing the spectrum, we performed an averaging over 10 pixels (3"' 6 ) along the slit to increase the signal-to-noise ratio for faint emission-line regions. The error boxes correspond to $3 \sigma$.

As follows from Fig. 8, the line intensity ratio $I([\mathrm{SII}]) / I(\mathrm{H} \alpha)$ increases significantly in the synchrotron superbubble region, thereby confirming the above comparison of the images in these lines. The value of $I([\mathrm{SII}]) / I(\mathrm{H} \alpha)=0.7-1.0$ observed in the superbubble strongly suggests the emission of the gas behind the shock front. The criterion $I([\mathrm{SII}]) / I(\mathrm{H} \alpha) \geq 0.6$ commonly used in revealing supernova remnants was exceeded significantly, given the low metallicity of IC $10, Z=0.2-0.3 Z$. (Skillman et al. 1989; Garnett 1990).

The distribution of the [SII] 6717/6731 A doublet line intensity ratio presented in Fig. 8c was used to determine the electron density of the gas in the synchrotron superbubble. In the [SII] line emission zone, we took $T_{\mathrm{e}}=10000 \mathrm{~K}$, a temperature typical of low-excitation zones. Taking into account the large observational error, we determined the density $n_{\mathrm{e}} \simeq 20-30 \mathrm{~cm}^{-3}$ only for the brighter northern part of the superbubble (the region $90^{\prime \prime}-$ $105^{\prime \prime}$ along the spectrogram), where the errors are relatively small.

\section{Discussion}

Our [SII] line images reveal the optical shell in the region of the synchrotron radio source much more clearly than the $\mathrm{H} \alpha$ images. The total size of the filamentary shell structure, about $44^{\prime \prime}$, corresponds to $168 \mathrm{pc}$. The synchrotron radio source has a comparable size: about $45-50^{\prime \prime}$, i.e., about $170-190 \mathrm{pc}$ at the distance of $790 \mathrm{kpc}$ assumed here (in Yang and Skillman (1993), the size of the radio source, $\geq 250 \mathrm{pc}$, corresponded to a distance of $1.25 \mathrm{Mpc})$.

The kinematics of the ionized gas in the galaxy was previously discussed by Bullejos and Rosado (2002), Rosado et al. (2002), and Thurow and Wilcots (2005). The first two papers are brief reports in conference proceedings; an expansion velocity of $50-70 \mathrm{~km} \mathrm{~s}^{-1}$ is only mentioned in them, which is completely confirmed by our observations. Thurow and Wilcots (2005) obtained $90 \mathrm{H} \alpha$ profiles for each of the five galaxy's fields, one of which covers part of the synchrotron superbubble. The authors present the radial velocity distribution (we completely confirmed these measurements) and point out a large line FWHM (about $90 \mathrm{~km} \mathrm{~s}^{-1}$ ) in the synchrotron superbubble region.

Our study of the gas kinematics in the synchrotron superbubble region is more systematic and detailed: we analyzed the position-velocity diagrams for more than 50 scans of different widths in different directions crossing the entire superbubble region and its immediate vicinities. As a result of this analysis, we determined the expansion velocity of the system of bright knots in the superbubble, $V_{\text {exp }}=50-80 \mathrm{~km} \mathrm{~s}^{-1}$ (the expansion velocity determined from weak line features is about $100 \mathrm{~km} \mathrm{~s}^{-1}$ ).

Based on our long-slit spectrum, we estimated the mean electron density of the gas in the northern part of the synchrotron superbubble from the [SII] doublet intensity ratio: $n_{\mathrm{e}} \simeq 20-30 \mathrm{~cm}^{-3}$. Thurow and Wilcots (2005) found a close value, $n_{\mathrm{e}}=30-50 \mathrm{~cm}^{-3}$, using the same method, but for a different orientation of the spectrograph slit.

Taking, as is commonly assumed in supernova remnants, the shell thickness to be about 0.1 of its radius, we can roughly estimate the mass of the optical shell and determine its kinetic energy from the expansion velocity found: $M \simeq 4 \times 10^{5} M_{\odot}$ and $E_{\mathrm{kin}} \simeq(1-3) \times 10^{52} \mathrm{erg}$, respectively. Thurow and Wilcots (2005) found a similar kinetic energy, $E_{\text {kin }}=(5-6) \times 10^{52} \mathrm{erg}$, from the mean $\mathrm{H} \alpha \mathrm{FWHM}$ in the superbubble. A lower kinetic energy of the optical shell, $E_{\text {kin }}=(0.6-1.2) \times 10^{51} \mathrm{erg}$, is mentioned in Bullejos and Rosado (2002) and Rosado et al. (2002), probably because these authors used an underestimated gas density.

As we noted above, the mass of the neutral gas showing signatures of the shell expansion with a velocity of $25 \mathrm{~km} \mathrm{~s}^{-1}$, reaches $7 \times 10^{5} M_{\odot}$, as estimated by Wilcots and Miller (1998). This gives a kinetic energy $E_{\text {kin }} \simeq$ $4 \times 10^{51} \mathrm{erg}$.

No more than $30 \%$ of the supernova explosion energy is known (see, e.g., Chevalier 1974) to be transferred to the ambient interstellar medium. Therefore, the total kinetic energy of the ionized and neutral gas found actually corresponds to the explosions of about ten supernovae, given that the stellar wind from the host association of these supernovae can provide approximately the same total kinetic energy input (see the estimates by Yang and Skillman 1993). Therefore, in present-day works (see, e.g., Bullejos and Rosado 2002; Rosado et al. 2002; Thurow and Wilcots 2005), based on the estimates of kinematic parameters for the ionized gas in the region of the nonthermal radio source, the authors also adopt the hypothesis of multiple supernova explosions. Bullejos and Rosado (2002) and Rosado et al. (2002) found a lower kinetic energy and estimated the necessary number of supernovae to be 3-6.

In (Lozinskaya and Moiseev 2007) we offered an alternative explanation for the nature of the synchrotron superbubble: a hypernova explosion. We pointed out that the binary X-ray source -1 , which is currently believed (Bauer and Brandt 2004; Wang et al. 2005) to be an accreting black hole in a pair with the WR star M17, is a possible compact remnant of this hypernova 1 .

We emphasize that the kinetic energy of the optical shell found here can be provided both by a hypernova ex-

1 Recently Prestwitch et al. (2007) and Silvereman and Filippenko (2008) showed the mass of the black-hole companion to M17 to be 23-35 solar masses and thereby fully confirmed the assumption of Lozinskaya and Moiseev (2007) concerning the Hypernova explosion. 


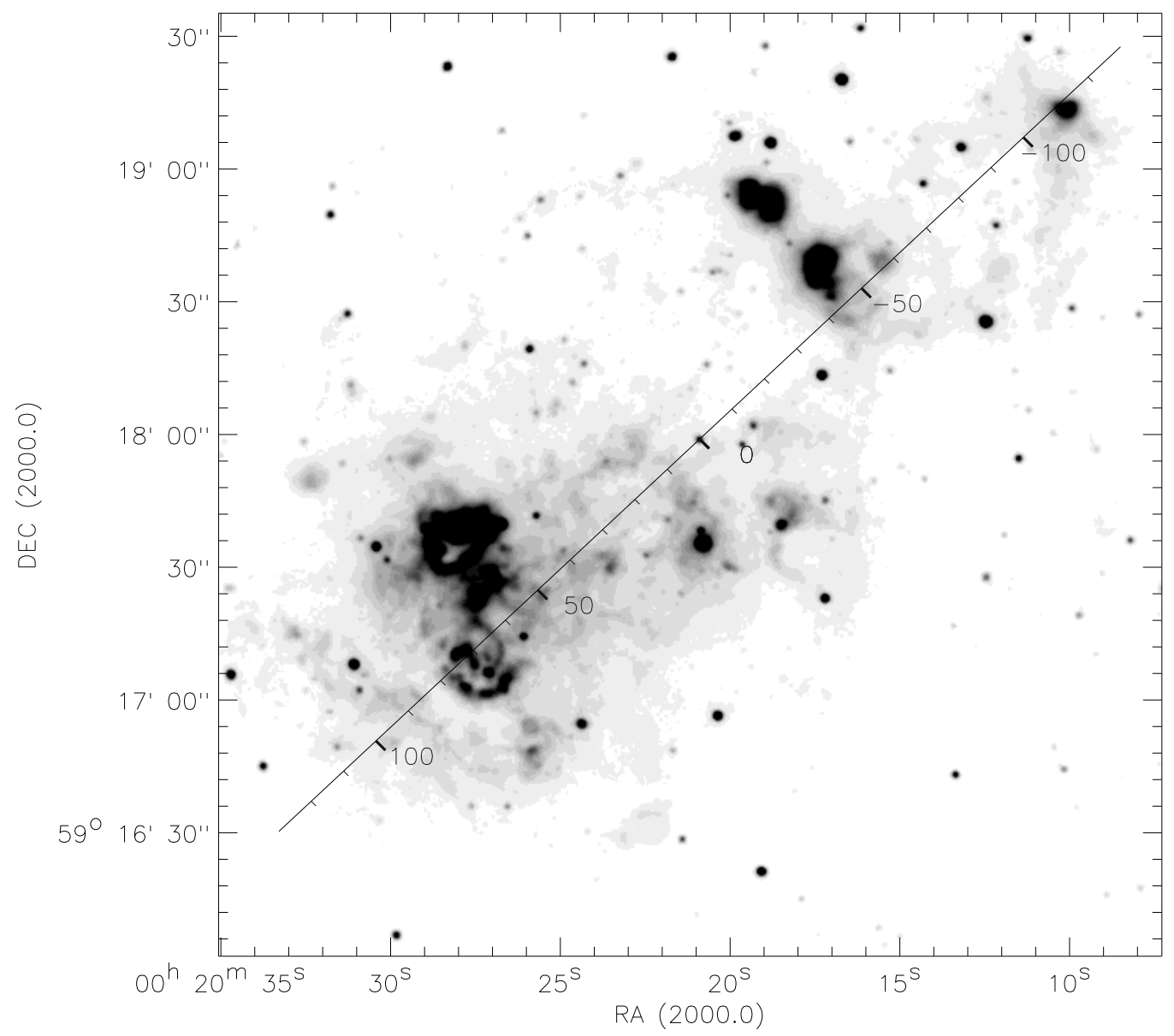

Fig. 7. Localization of the slit spectrogram passing through the synchrotron superbubble (image in $\mathrm{H} \alpha+$ continuum); the spectrogram is marked up in arsec.

plosion and by the model of multiple supernova explosions suggested previously. In (Lozinskaya and Moiseev 2007), we reached the conclusion that a hypernova explosion explained better the nature of the synchrotron superbubble than multiple supernova explosions by analyzing the radio emission from the object. In this paper, for the first time, we have taken into account the fact that the explosions of "recent" supernovae in the model by Yang and Skillman (1993) occur in a tenuous cavity inside the superbubble swept out by the "first" supernovae. Our analysis of this effect (Lozinskaya and Moiseev 2007) showed that subsequent supernova explosions add little to the radio brightness of the synchrotron superbubble produced by the first explosions and, accordingly, the necessary number of explosions in the model of multiple supernovae increases significantly. Therefore, a hypernova explosion seems a more plausible formation mechanism of the synchrotron superbubble.

In recent years, interest in the possible hypernova remnants has increased greatly, because the latter are assumed to be associated with gamma-ray bursts. However, the kinetic energy of the superbubble found $E_{0}=(1-$ 10) $\times 10^{52}$ erg can often be adequately explained not only by a hypernova explosion, but also in terms of the classical model of the combined action of stellar winds and multiple supernovae of an OB association. Indeed, multiple supernova explosions in the galaxy's local region suggest the presence of a rich host OB association. The results of a detailed analysis of the physical conditions and nature of the two presumed hypernova remnants in the galaxy M 101 performed by Lai et al. (2001) and Chen et al. (2002) can serve as an illustration. One of them (MF 83) can be reliably reidentified as the superbubble swept out and ionized by the stars of four OB associations; the second (NGC 5471) can actually be associated with a hypernova explosion. Note that the object MF 83 is not associated with the synchrotron radio source, which should have immediately called into question its identification as being the result of a hypernova explosion or multiple supernova explosions. However, this question is not quite unambiguous either: the presence of a gas ionized by the host association can make the thermal radio emission in the region predominant.

The anomalously high kinetic energy of some HI shells surrounding classical supernova remnants (see, e.g., Gosachinskij and Khersonskij (1985), Gosachinskij (2005), and references therein) do not provide unequivocal evidence for a hypernova explosion either. First, the wind 

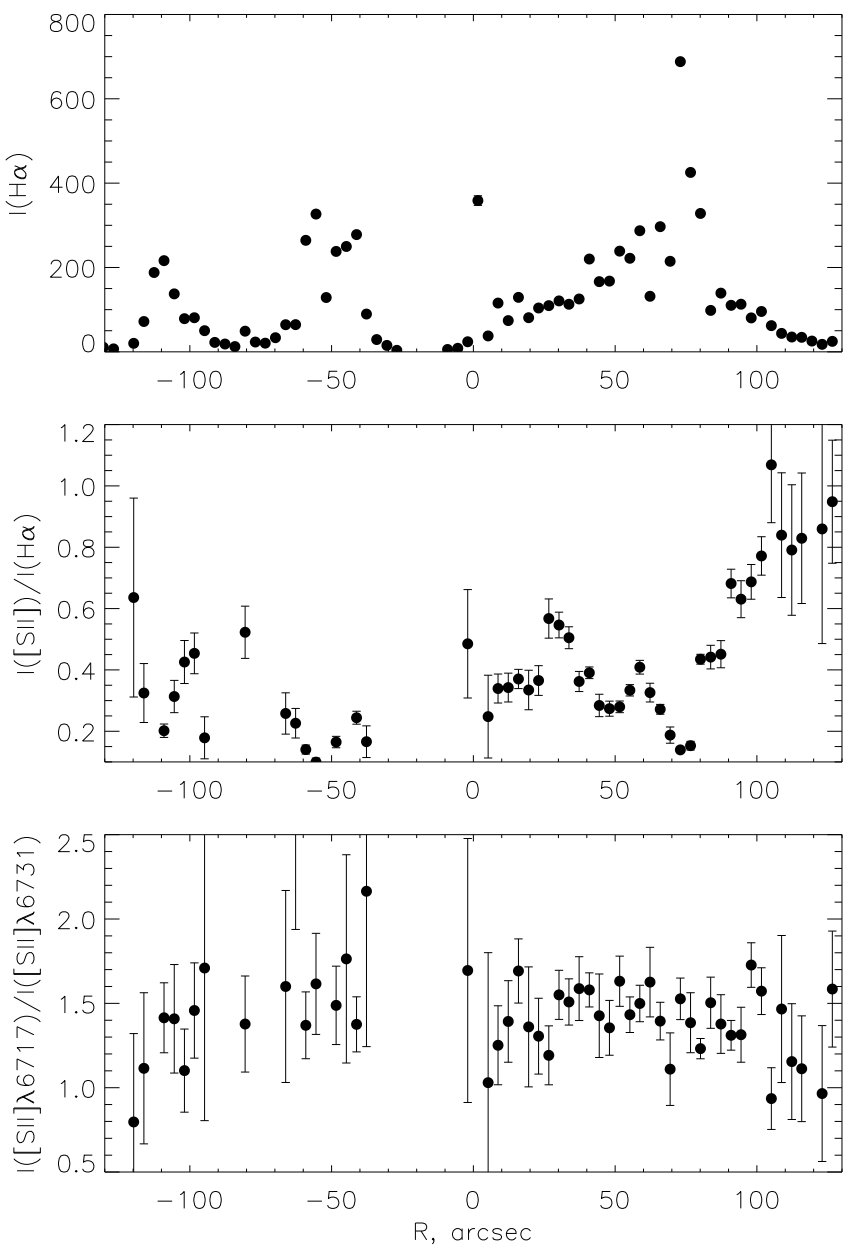

Fig. 8. The spectrogram processing results: top $\mathrm{H} \alpha$ intensity distribution along the slit; middle line intensity ratio $I([\mathrm{SII}] \lambda(6717+6731) \AA) / I(\mathrm{H} \alpha)$; bottom [SII] doublet line intensity ratio $I(\lambda 6717 \AA) / I(\lambda 6731 \AA)$. The region from $90^{\prime \prime}$ to $125^{\prime \prime}$ corresponds to the synchrotron superbubble.

from the supernova progenitor and/or the stars of the host $\mathrm{OB}$ association is an obvious energy source of the outer HI shell. Second, the passage from the estimated kinetic energy of the HI shell to the initial supernova explosion energy is model-dependent.

Therefore, the main criterion that allows the superbubble in IC 10 investigated here to be reliably considered a remnant of hypernova explosion is its strong synchrotron radio emission, which, as we showed in (Lozinskaya and Moiseev 2007), is in better agreement with a hypernova explosion than with multiple supernova explosions.

The age of the superbubble determined by its size and the expansion velocity found, $50-80 \mathrm{~km} \mathrm{~s}^{-1}$, is $t \simeq(4-$ $7) \times 10^{5} \mathrm{yr}$, if the remnant is at the Sedov stage or $t \simeq(3-$ $5) \times 10^{5}$ yr for the radiative cooling stage.

This age is a strong argument for the hypothesis of a hypernova explosion. It takes at least $t \simeq 10^{7} \mathrm{yr}$ for the explosions of about ten supernovae in the galaxy's local region about $200 \mathrm{pc}$ in size.

Note also that the explosions of about ten supernovae in a short time interval suggest the presence in the region of a rich association of young stars (up to 800 massive stars with $M \geq 10 M_{\odot}$, similar to 30 Dor and R136, as estimated by Yang and Skillman (1993)). It is hard to "hide" this rich host association, although it cannot be completely ruled out in principle, given the strong absorption due to the low Galactic latitude of IC 10 and the location of the synchrotron superbubble near the densest dust layer in IC 10. Note that Hunter (2001) identified two clusters near the southern boundary of the superbubble, 4- 6 and 4-7 in her notation, but their colors were not determined and there is no age estimate. These are by no means richest clusters in the galaxy and they are definitely not suitable for the role of the "hosts" for the hypothetical multiple supernovae.

In the plane of the sky, the synchrotron superbubble is located in the region of the densest HI cloud in the galaxy; the densest CO cloud in IC 10 also coincides with the core of this cloud (see Fig. 4 from Leroy et al. 2006).

Comparison of the ionized and neutral gas kinematics in the synchrotron superbubble region reveals a clear "asymmetry" in the HII and HI velocity distribution. Indeed, the most distinct "high-velocity" $\mathrm{H} \alpha$ emission features are shifted toward the negative velocities, while the relatively more amorphous $\mathrm{HI}$ emission regions represent the receding side of the expanding shell at the velocities shifted toward the positive values. Based on the observed "asymmetric" kinematics of the ionized and neutral gas, we conclude that along the line of sight, the synchrotron superbubble is located on the near side of a dense cloud. The absence of clear morphological signatures of the HI shell structure in the vicinity of the synchrotron superbubble noted above most likely stems from the fact that only a small fraction of the HI column density is involved in this expansion that is clearly revealed by its kinematics.

The fact that the neutral gas reveals a classical velocity ellipse, while the ionized gas reveals only separate, rapidly moving knots can be explained by the homogeneity of the $\mathrm{HI}$ region and by the clumpiness of the HII region, which is clearly seen in the $[\mathrm{SII}]$ and $\mathrm{H} \alpha$ line images presented above.

\section{Conclusions}

Our [SII] line image reveals the optical shell about $170 \mathrm{pc}$ in size (at a distance of $790 \mathrm{kpc}$ ) that can be identified with the synchrotron superbubble better than the $\mathrm{H} \alpha$ image.

A detailed study of the ionized gas kinematics allowed us to estimate the characteristic expansion velocity of the bright knots and filaments in the optical shell: 50$80 \mathrm{~km} \mathrm{~s}^{-1}$; the expansion velocity determined from weak line features is $\sim 100 \mathrm{~km} \mathrm{~s}^{-1}$.

Having estimated the electron density in the northern part of the optical shell from the $[\mathrm{SII}] 6717 / 6731 \AA$ ratio, $n_{\mathrm{e}} \simeq 20-30 \mathrm{~cm}^{-3}$, by assuming the shell thickness to be $\sim 0.1$ of its radius, we found its mass $M \simeq 4 \times 10^{5} M_{\odot}$ and kinetic energy $E_{\text {kin }} \simeq(1-3) \times 10^{52} \mathrm{erg}$ from the measured expansion velocity of $50-80 \mathrm{~km} \mathrm{~s}^{-1}$. This energy is intermediate between the estimates $E_{\mathrm{kin}} \simeq\left(5^{-}\right.$ 
6) $\times 10^{52}$ erg from Thurow and Wilcots (2005) and $E_{\text {kin }} \simeq$ $(0.6-1.2) \times 10^{51}$ erg from Bullejos and Rosado (2002) and Rosado et al. (2002). The shell's energy found corresponds to the explosions of about ten supernovae plus the stellar wind from their host association, as suggested by Yang and Skillman (1993), or a hypernova explosion, as we suggested in (Lozinskaya and Moiseev 2007).

The shell age, $t \simeq(3-7) \times 10^{5} \mathrm{yr}$, corresponding to the derived expansion velocity is a strong argument for the hypothesis of a hypernova explosion, since it takes at least $t \simeq 10^{7} \mathrm{yr}$ for the explosions of ten supernovae in the galaxy's local region.

Comparison of the ionized and neutral gas kinematics leads us to conclude that the synchrotron superbubble is located on the near side of a dense $\mathrm{HI}$ and $\mathrm{CO}$ cloud observed here.

Acknowledgements. This work was supported by the Russian Foundation for Basic Research (project nos. 05-02-16454 and 07-02-00227). The work is based on the observational data obtained with the 6-m SAO RAS telescope funded by the Ministry of Science of Russia (registration no. 01-43). We thank A. Valeev for his help in the observations and E. Wilcots, who provided the data cube of HI observations. When working on the paper, we used the NASA/IPAC Extragalactic Database (NED) operated by the Jet Propulsion Laboratory of the California Institute of Technology under contract with the National Aeronautics and Space Administration (USA).

\section{References}

V. L. Afanasiev and A. V. Moiseev, Astron. Lett. 31, 194 (2005); astro-ph/0502095

F. E. Bauer and W. N. Brandt, Astrophys. J. 601, L67 (2004).

W. D. Brandt, M. J. Ward, A. C. Fabian, and P. W. Hodge, Mon. Not. R. Astron. Soc. 291, 709 (1997).

A. Bullejos and M. Rosado, Rev. Mex. Astron. Astrofis. (Ser. Conf.) 12, 254 (2002).

C.-H. R. Chen, You-Hua Chu, R. Gruendl, and Sh.-P. Lai, Astron. J. 123, 2462 (2002).

R. A. Chevalier, Astrophys. J. 188, 501 (1974).

K. T. Chyzy, J. Knapik, D. J. Bomans, et al., Astron. Astrophys. 405, 513 (2003).

P. A. Crowther, L. Drissen, J. B. Abbott, et al., Astron. Astrophys. 404, 483 (2003).

S. Demers, P. Battinelli, and B. Letarte, Astron. Astrophys. 424, 125 (2004).

D. R. Garnett, Astrophys. J. 363, 142 (1990).

A. Gil de Paz, B. F. Madore, and O. Pevunova, Astrophys. J., Suppl. Ser. 147, 29 (2003).

I. V. Gosachinskii, Astron. Lett. 31, 179 (2005).

I. V. Gosachinskij and V. K. Khersonskij, Astrophys. Space Sci. 108, 303 (1985).

D. A. Hunter, Astrophys. J. 559, 225 (2001).

Sh.-P. Lai, You-Hua Chu, and C.-H.R. Chen, Astrophys. J. 547, 754 (2001).

A. Leroy, A. Bolatto, F. Walter, and L. Blitz, Astrophys. J. 643, 825 (2006).

T. A. Lozinskaya and A. V. Moiseev, Mon. Not. R. Astron. Soc. 381, L26 (2007).

P. Massey and S. Holmes, Astrophys. J. 580, L35 (2002).
P. Massey, T. E. Armandroff, and P. S. Conti, Astron. J. 103, 1159 (1992).

P. Massey, K. Olsen, P. Hodge, et al., Astron. J. 133, 2393 (2007).

A. V. Moiseev, Bull. Spec. Astrophys. Obs. 54, 74 (2002); astro-ph/0211104

A. H. Prestwich, R. Kilgard, P. A. Crowther et al. Astroph.J., 669, L21 (2007)

M. G. Richer, A. Bullejos, J. Borissova, et al., Astron. Astrophys. 370, 34 (2001).

M. Rosado, A. Bullejos, M. Valdez-Gutierrez, et al., in Proceedings of the IAU Symposium 190: New Views of the Magellanic Clouds, Ed. by Y.-H. Chu et al. (Astron. Soc. Pac., San-Francisco, 1999), p. 168.

M. Rosado, M. Valdez-Gutierrez, A. Bullejos, et al., Astron. Soc. Pac. Conf. Ser. 282, 50 (2002).

P. Royer, S. J. Smart, J. Manfroid, and J. Vreux, Astron. Astrophys. 366, L1 (2001).

J. M. Silverman, A. V. Filippenko, ApJL, submitted (2008); arXiv:0802.2716 [astro-ph]

E. D. Skillman, R. C. Kennicutt, and P. W. Hodge, Astrophys. J. 347, 875 (1989).

J. C. Thurow and E. M. Wilcots, Astron. J. 129, 745 (2005).

W. D. Vacca, C. D. Sheehy, and J. R. Graham, Astrophys. J. 662, 272 (2007).

Q. D. Wang, K. E. Whitaker, and R. Williams, Mon. Not. R. Astron. Soc. 362, 1065 (2005).

E. M. Wilcots and B. W. Miller, Astron. J. 116, 2363 (1998).

H. Yang and E. D. Skillman, Astron. J. 106, 1448 (1993).

D. B. Zucker, Bull. Am. Astron. Soc. 32, 1456 (2000).

D. B. Zucker, Bull. Am. Astron. Soc. 34, 1147 (2002).

Translated by V. Astakhov 